changed. Rates of wrapping, residua, and regrowth were not statistically different based on aneurysm rupture status.

Conclusion Open surgery for cerebral aneurysm results in incomplete treatment approximately $12 \%$ of the time. This should be taken into account when considering endovascular verses open surgical therapy and counseling patients. These results should be interpreted with caution considering the significant amount of heterogeneity between the included studies.

Disclosures D. Schartz: None. T. Mattingly: None. T. Bhalla: None. M. Bender: None.

\section{P-046 FACTORS ASSOCIATED WITH INCOMPLETE OCCLUSION OF INTRACRANIAL ANEURYSMS AT FOLLOW UP AFTER TREATMENT WITH WOVEN ENDOBRIDGE (WEB) DEVICE}

K Javed ${ }^{*}$, A Fortunel, N Haranhalli, D Altschul. Neurosurgery, Montefiore Medical Center, Bronx, NY

\subsection{6/neurintsurg-2021-SNIS.82}

Objective The Woven EndoBridge (WEB) device is a novel treatment option for wide-necked bifurcation intracranial aneurysms (WBNA), which historically were a challenge to treat using conventional endovascular therapies. According to the WEB-IT study which led to the WEB device gaining FDA approval in the US, the complete aneurysmal occlusion rate is $53.8 \%$ while the adequate occlusion rate is $84.6 \%$ at one year follow up. While this device has had good results, there remains a subset of WBNA that fail this treatment. The main objective of this study is to identify factors that are associated with incomplete occlusion of WEB treated aneurysms at follow up.

Methods This is a retrospective study of forty-one patients with intracranial aneurysms who were treated with WEB placement at a single institution since 2019. Data was collected via electronic medical record (EMR) review on patient demographics, medical comorbidities, aneurysm characteristics, procedural details, and occlusion status at six months follow up. Bivariate analyses were performed comparing completely occluded aneurysms with incompletely occluded aneurysms such as neck remnants and residual aneurysms.

Results Of the 41 patients, follow up data was only available for 25 patients. $13(52 \%)$ of those 25 patients had completely occluded treated aneurysms while $8(32 \%)$ patients had a neck remnant and $4(16 \%)$ patients had a residual aneurysm at six months follow up. On bivariate analysis, patients with a neck remnant were found to have a median angle of $29.7^{\circ}$ in the true neck view compared to a median angle of $17.7^{\circ}$ in patients with complete occlusion $(p$ value $=0.050)$. In patients with residual aneurysms, the median angle in the down the barrel view was $9.3^{\circ}$ while it was $34.2^{\circ}$ in patients with occluded aneurysms ( $p$ value $=0.023$ ). Furthermore, the average diameter of the WEB device deployed was $7.5 \mathrm{~mm}$ in patients with residual aneurysms compared to $6.0 \mathrm{~mm}$ in the complete occlusion group ( $\mathrm{p}$ value $=0.026$ ). Lastly, patients with residual aneurysms were more likely to have a longer procedure time $(p$ value $=0.027$ ) and less likely to have an immediate angiographic outcome of complete occlusion ( $p$ value $=0.006$ ) .

Conclusion The angular measurements of the aneurysms along with size of the WEB device used, procedure length and immediate post-treatment occlusion status may predict the occlusion status of the treated aneurysm at six months follow up.

Disclosures K. Javed: None. A. Fortunel: None. N. Haranhalli: None. D. Altschul: None.

\section{P-047 MULTIMODAL ENDOVASCULAR TREATMENT OF WIDE- NECKED ANEURYSMS: SAFETY AND OCCLUSION OUTCOMES FROM THE STERLING REGISTRY}

${ }^{1} \mathrm{R}$ De Leacy* ${ }^{*}{ }^{2} \mathrm{~A}$ Puri, ${ }^{3} \mathrm{R}$ Starke, ${ }^{4} \mathrm{~B}$ Jankowitz, ${ }^{5} \mathrm{~A}$ Yoo, ${ }^{6} \mathrm{G}$ Pero, ${ }^{7} \mathrm{C}$ Chivot, ${ }^{8} \mathrm{~T}$ Yao, ${ }^{9} \mathrm{O}$ Zaidat. ${ }^{1}$ Neurosurgery, Mount Sinai Health System, New York, NY; ${ }^{2}$ Radiology, Neurological Surgery, and Neurosurgeny, UMass Memorial Health Care, Boston, MA; ${ }^{3}$ Neurosurgery, UMiamilJackson Memorial, Miami, FL; ${ }^{4}$ Neurological Institute, Neurological Surgery, and Stroke Program, Cooper University Health Care, Pittsburgh, PA; ${ }^{5}$ Neurological Surgery, Texas Stroke Institute, Plano, TX; ${ }^{6}$ Neuroradiology, Ospedale Niguarda, Milan, Italy;

${ }^{7}$ Radiology, CHU Amiens Picardie, Amiens, France; ${ }^{8}$ Neurosurgery, Norton Neuroscience Institute, Norton Healthcare, Louisville, KY; ${ }^{9}$ Neuroscience Institute, Mercy St. Vincent Hospital, Toledo, $\mathrm{OH}$

\subsection{6/neurintsurg-2021-SNIS.83}

Purpose We present core-lab adjudicated immediate post-procedural angiographic outcomes following endovascular treatment of side wall and bifurcation wide-necked aneurysms using MICRUSFRAME and GALAXY coils and various adjunctive devices within the STERLING registry.

Methods STERLING is an ongoing single-arm prospective post-market surveillance registry evaluating ruptured/unruptured aneurysms treated with MICRUSFRAME and GALAXY coils (Cerenovus, Irvine, CA). All cases of wide-neck aneurysm treatment (neck $\geq 4 \mathrm{~mm}$ or dome-to-neck ratio $<2$ ) were extracted from the registry. Subgroups were defined according to the adjunctive devices (if any) used during treatment. The primary outcome measures were core-lab adjudicated modified Raymond-Roy (mRR) occlusion at final procedural angiogram, and procedure-related adverse events.

Results The study cohort contained 168 patients treated for 168 wide-necked aneurysms. The average age was 59.1 (SD 10.5 ), and $71.4 \%$ of patients were female. Previously treated aneurysms accounted for $9.5 \%$ of the cohort, and $45(26.8 \%)$ were acutely ruptured. All acutely ruptured aneurysms were treated with coiling alone. One hundred thirty-seven aneurysms $(81.5 \%)$ were located in the anterior circulation, and 90 $(53.6 \%)$ were located at a bifurcation point. With regard to size, $22.6 \%$ of aneurysms were $<5 \mathrm{~mm}$ in diameter, $71.4 \%$ were $\geq 5 \mathrm{~mm}$ and $<13 \mathrm{~mm}$, and the remaining $6.0 \%$ were $\geq$ $13 \mathrm{~mm}$. The average procedure time was 1.74 hours (SD 0.99 ) and the average fluoroscopy time was 49.7 minutes (SD 29.6).

Aneurysms were treated using the following modalities: coiling alone (58.3\%), stent-assisted coiling (24.4\%), coiling plus flow diversion (8.3\%), Pulserider-assisted coiling (4.2\%), balloon-assisted coiling (1.2\%), other adjunctive devices (1.8\%), and multiple adjunctive devices (1.8\%). Across the entire cohort, $82.1 \%$ of patients achieved adequate occlusion, defined as mRR class I or II. Angiographic outcomes according to treatment modality are summarized in table 1.

Procedure-related adverse events occurred in 7 cases (4.2\%). These included 3 intraprocedural ruptures (1 ruptured, 2 unruptured at baseline), 1 cerebrovascular accident, and 3 asymptomatic thromboembolic events. There were no deaths. Conclusion Endovascular treatment of wide-neck aneurysms using MICRUSFRAME and GALAXY coils is safe and effective 